\title{
Cellular Uptake of IgG Using Collagen-Like Cell-Penetrating Peptides
}

\author{
Ryo Masuda, ${ }^{*, a, b}$ Kazuhiro Yamamoto, ${ }^{a}$ and Takaki Koide ${ }^{a}$ \\ ${ }^{a}$ Department of Chemistry and Biochemistry, School of Advanced Science and Engineering, Waseda University; \\ Tokyo 169-8555, Japan: and ${ }^{b}$ Research Institute for Science and Engineering, Waseda University; Tokyo 169-8555, \\ Japan. \\ Received July 9, 2015; accepted October 19, 2015
}

Cell-penetrating peptides (CPPs) are attractive tools for delivering macromolecules that have poor membrane permeability, such as antibodies, into cells. However, the major drawback of conventional CPPs is their instability in bodily fluids. We previously reported a novel CPP employing a collagen-like triple-helical structure that exhibited remarkable resistance against serum proteases. Herein, we report the delivery of full-length immunoglobulin G (IgG) antibody into cells using a triple-helical CPP. The CPP was conjugated to IgG via a one-pot reaction using 2 -iminothiolane as a crosslinking reagent. The triple-helical CPP was less prone to being aggregated and neutralized by serum than was octaarginine, a conventional CPP. However, most of the conjugates were found to be entrapped in endosomes.

Key words cell-penetrating peptide; antibody delivery; collagen-like peptide; peptide conjugation

Antibody drugs have many clinical applications including cancer and rheumatoid arthritis therapy. ${ }^{1)}$ The hallmark of antibody drugs is their high specificity toward target molecules, which helps to limit their side effects compared with conventional small molecular drugs. In contrast, because of their poor cellular membrane permeability, the targets of antibody drugs are currently limited to extracellular molecules. ${ }^{2}$

Cell-penetrating peptides (CPPs) have been broadly used in vitro as a carrier to deliver cargoes into cells. Most are rich in basic amino acid residues, which allows them to interact with negatively charged glycosaminoglycan chains on the cellular membrane, followed by uptake into cells majorly via endocytosis/micropinocytosis., ${ }^{3,4)}$ Efficient delivery of large molecules such as antibodies into cells by CPPs requires 1) accumulation of the molecules into endosomes and 2) release of the molecules from the endosomes. However, the instability of CPPs in bodily fluids is one of the major drawbacks that limits their in vivo use. ${ }^{5)}$ In addition, although intracellular delivery of antibody drugs by CPPs has been attempted to date, the reported methods were not sufficiently practical because of requirement of multistep workups, ${ }^{6}$ and demand for too high concentration of CPP. ${ }^{7)}$ The success in efficient conjugation of CPP by chemical procedure was restricted to application to single-chain variable fragment because of usage of free thiols. ${ }^{8)}$ Although another approach for the conjugation of CPP to amine groups in Fab fragment was reported, the conjugation precursor needed to be prepared. ${ }^{9)}$ Therefore, the development of facile and efficient method to conjugate CPP to full length immunoglobulin $\mathrm{G}$ ( $\mathrm{IgG}$ ) is required for both efficient endosome targeting and release from endosomes.

Recently, we developed Arg-rich triple-helical CPPs that mimicked the rigid rod-like structure of collagen. ${ }^{10)}$ The triple-helical CPPs were shown to have remarkable stability in serum with low binding to serum proteins, implying their in vivo appliations. ${ }^{10,11)}$ Herein, we report the strategy for conjugating the CPP to $\operatorname{IgG}$, properties of the complexes, and its delivery and intracellular localization.

\section{MATERIALS AND METHODS}

Peptide Synthesis and Characterization The peptide chains of colpep (H-(Gly-Pro-Hyp) $)_{3}$-(Gly-Pro-Arg) $)_{3}$-(GlyPro-Hyp) ${ }_{3}$-Gly-Pro- $\mathrm{NH}_{2}$ (Hyp: L-4-hydroxyproline)) and $\boldsymbol{\beta} \mathbf{R 8}$ (H- $\beta$ Ala-(Arg) $)_{8}-\mathrm{NH}_{2}$ ( $\beta$ Ala: $\beta$-alanine)) were constructed using the 9-fluorenylmethyloxycarbonyl-based solid-phase method on Rink-amide resin (Novabiochem, Nottingham, U.K.). For preparation of N-terminally iodoacetylated peptide, peptide resins were reacted with iodoacetic acid (5eq) in the presence of $N, N^{\prime}$-diisopropylcarbodiimide (5 eq) and 1-hydroxybenzotriazole (5eq) in $N, N$-dimethylformamide for $2 \mathrm{~h}$ at room temperature. Purification and measurement of circular dichroism (CD) spectra were performed according to the previous report. ${ }^{10)}$

Conjugation of CPPs to IgG Triple-helical CPP was treated at $95^{\circ} \mathrm{C}$ for $5 \mathrm{~min}$, followed by incubation at $4^{\circ} \mathrm{C}$ overnight to form triple-helix. Fluorescein isothiocyanate (FITC)-labeled $\alpha$-rabbit goat $\operatorname{IgG}(8 \mu \mathrm{M}$, Life Technologies, Gaithersburg, MD, U.S.A.) was reacted with 2-iminothiolane hydrochloride and iodoacetylated peptide at the described concentration in $4 \mathrm{~mm}$ triethanolamine hydrochloride buffer $(\mathrm{pH} 8.5)$ containing ethylenediaminetetraacetic acid $(100 \mu \mathrm{M})$ at room temperature for $20 \mathrm{~h}$. The reaction mixture was fractionated through Sephadex G-25 on a PD10 column (GE Healthcare, Chalfont, St. Giles, U.K.) with phosphate buffered saline (PBS), and concentrated by Amicon Ultra $30 \mathrm{kDa}$ cutoff filter $(0.5 \mathrm{~mL}$, Millipore, Billerica, MA, U.S.A.). The peptide-labeling to IgG was confirmed by sodium dodecyl sulfate-polyacrylamide gel electrophoresis (SDS-PAGE) using a $10-20 \%$ gradient gel.

Delivery of Complexes into Cells For confocal microscopic analysis, HeLa cells $\left(2.0 \times 10^{4}\right.$ cells $)$ were plated on 35-mm glass-bottomed dishes (Iwaki, Chiba, Japan) and cultured in Dulbecco's modified Eagle's medium (DMEM) containing $10 \%$ fetal bovine serum (FBS) for $1 \mathrm{~d}$. Cells were then subjected to IgG-CPP complexes $(40 \mu \mathrm{g} / \mathrm{mL})$ in DMEM for $3 \mathrm{~h}$ at $37^{\circ} \mathrm{C}$. The resulting cells were washed with DMEM containing $0.5 \mathrm{mg} / \mathrm{mL}$ heparin sodium (Nacalai Tesque, Kyoto, 
a)

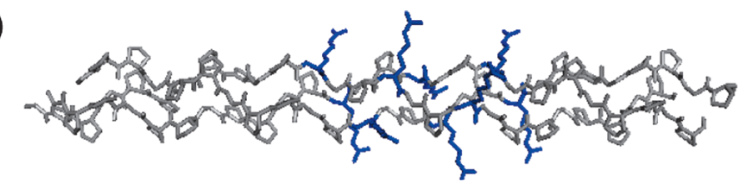

b)

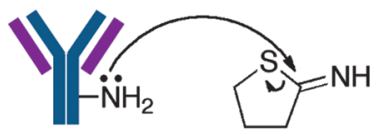

antibody

2-iminothiolane<smiles>O=C(CI)NC1CCCC1</smiles>

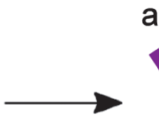

$1 / \mathrm{H}$<smiles>CCCCC(C)=N</smiles>

$\longrightarrow 1$<smiles>CCCCNC(=N)Nc1ccccc1C</smiles>

CPP: - $(\mathrm{GPO})_{3}(\mathrm{GPR})_{3}(\mathrm{GPO})_{3} \mathrm{GP}$-amide (colpep) $-\beta A R_{8}$-amide ( $\beta$ R8)

c)

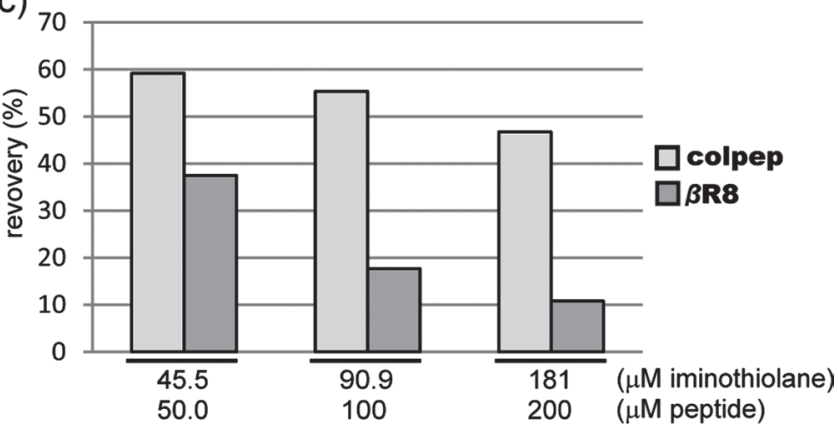

Fig. 1. Conjugation of Triple-Helical CPP to IgG

(a) A 3D model of colpep trimer was created by processing the PDB file $1 \mathrm{k} 6 \mathrm{f}$ using Pymol software. Arg residues are shown in blue. (b) Synthetic scheme for CPP-conjugated IgG. (c) Recovery of IgG after the conjugation reaction.

Japan) and observed using a FV1000 confocal microscope (Olympus, Tokyo, Japan). For the staining of endosome and lysosome, tetramethylrhodamine (TRITC)-conjugated transferrin (Life Technologies) and LysoTracker Red DND-99 (Life Technologies) were used according to the manufacturer's protocol, respectively.

For fluorescence-activated cell sorting (FACS) analysis, HeLa cells were treated with $\mathrm{IgG}-\mathrm{CPP}$ complexes $(40 \mu \mathrm{g} / \mathrm{mL})$ in DMEM for $3 \mathrm{~h}$ at $37^{\circ} \mathrm{C}$. The cells were washed with PBS containing $0.5 \mathrm{mg} / \mathrm{mL}$ heparin sodium, and then detached by the treatment with $0.05 \%$ trypsin. The collected cells were pelleted by centrifugation at $800 \times \boldsymbol{g}$ for $3 \mathrm{~min}$, and the cells washed with PBS. The resultant cells were analyzed by a $\mathrm{Cy}$ tomics FC 500 flow cytometer (Beckman Coulter, Miami, FL, U.S.A.).

\section{RESULTS}

We employed the 29-mer peptide colpep, which has three Gly-Pro-Arg guest sequences flanked by three repeats of GlyPro-Hyp at the N- and C-termini to stabilize the triple-helical structure, as the test CPP. As shown by the 3D model in Fig. 1a, a cluster of Arg residues formed in the central region of the triple helix. Octaarginine with an N-terminal $\beta$-alanine linker $(\boldsymbol{\beta} \mathbf{R 8})$ was also synthesized as a control. We utilized a)

FITC-IgG

DIC/overlay

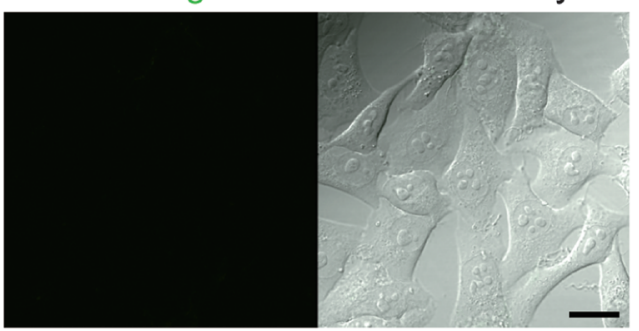

IgG-colpep

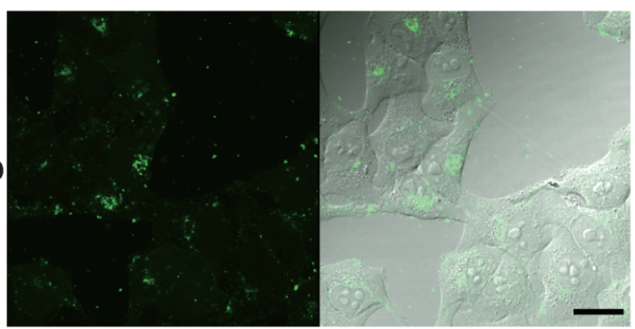

IgG- $\beta$ R8

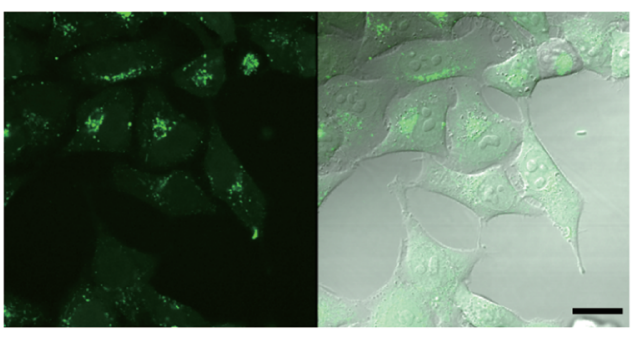

b)

C)
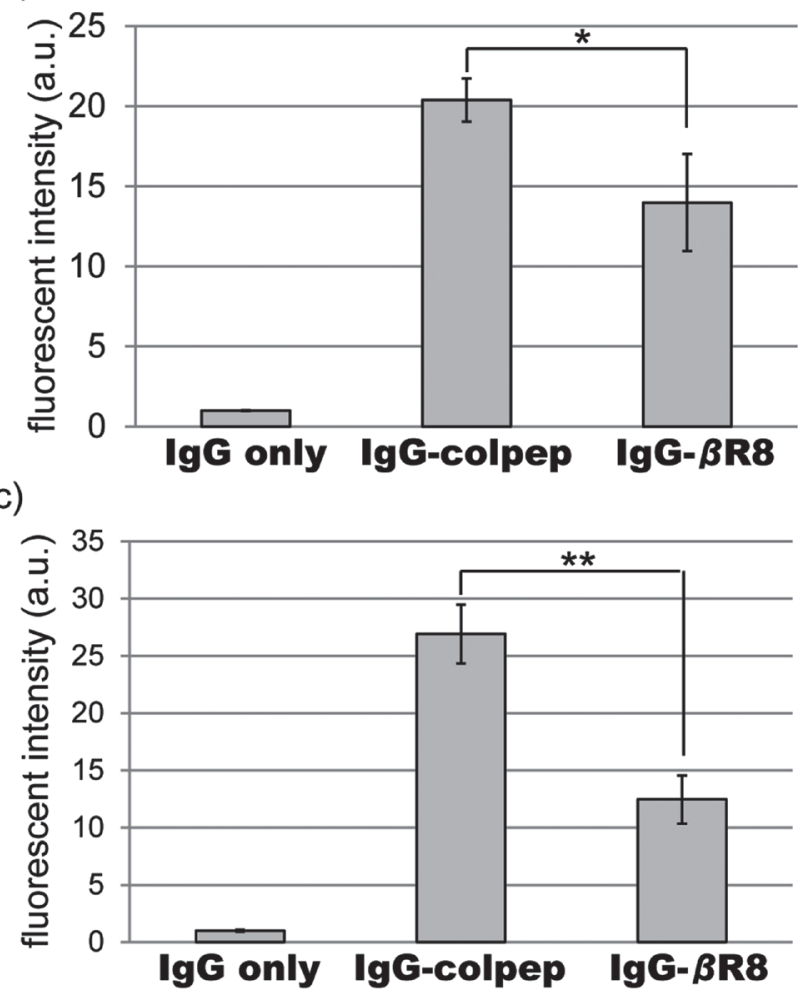

Fig. 2. Cellular Uptake of IgG-CPP Complexes

(a) Fluorescence images obtained using a confocal microscope. Scale bar is $20 \mu \mathrm{m}$. DIC: differential interference contrast. (b, c) Quantitative analysis using flow cytometry of IgG delivered into cells without (b) or with (c) pre-treatment of FBS ( 1 as IgG only. mean \pm S.D., $n=3 ; * p<0.1, * * p<0.01$ ).

the $\varepsilon$-amino groups on $\mathrm{IgG}$ for conjugation with the CPPs. N-terminally iodoacetylated peptides, iodoacetyl-colpep or iodoacetyl- $\boldsymbol{\beta}$ R8, were reacted with FITC-IgG in the pres- 
a)

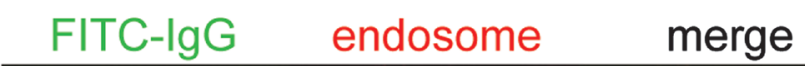

IgG only
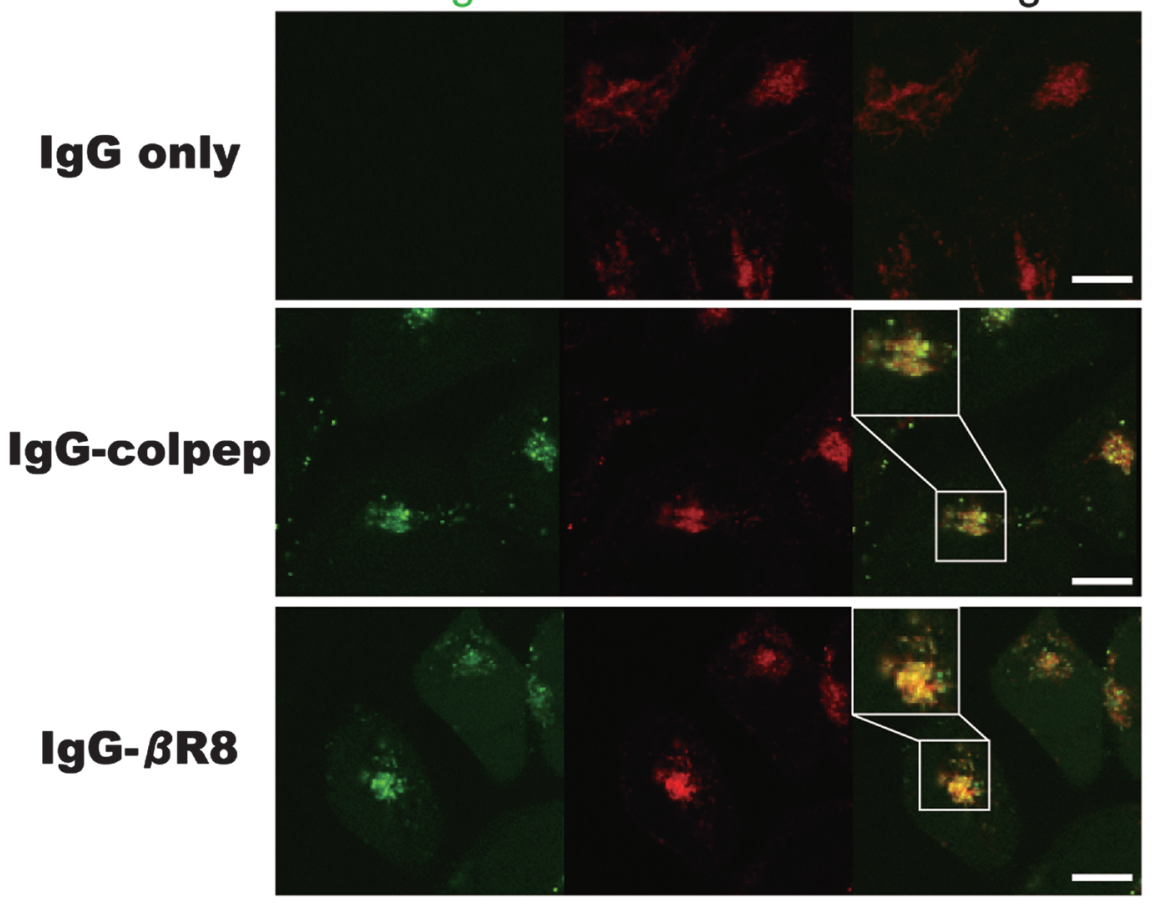

b)

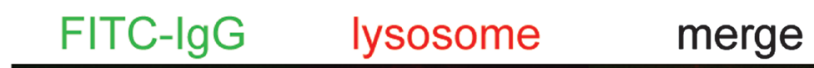

IgG-colpep

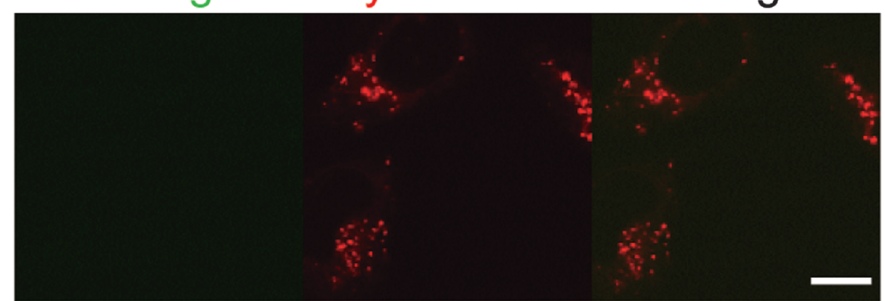

IgG only
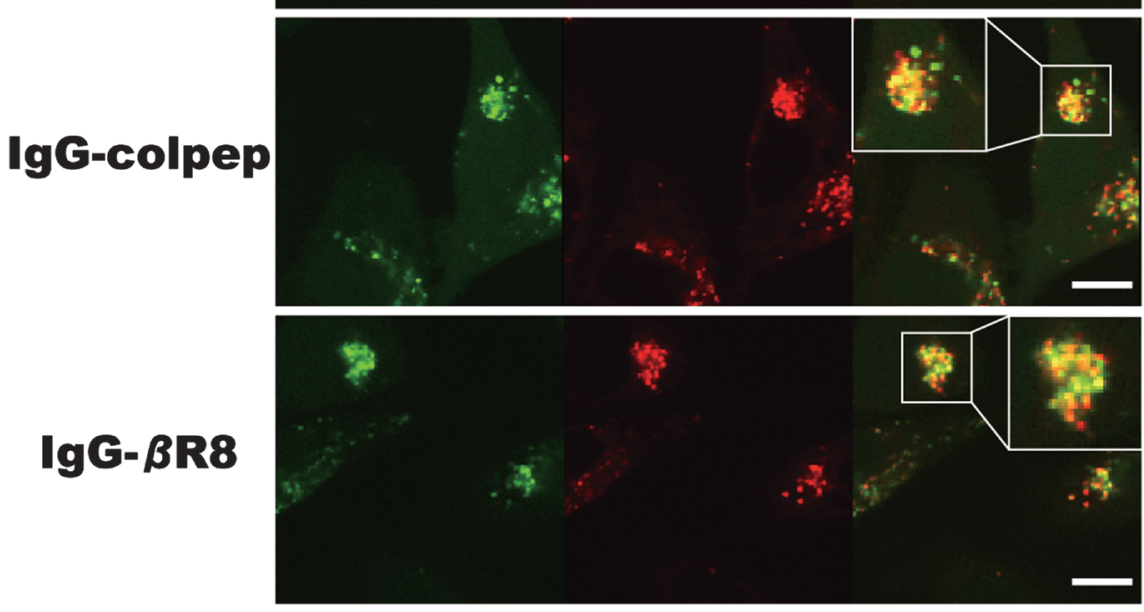

Fig. 3. Intracellular Localization of $\mathrm{IgG}-\mathrm{CPP}$ Complexes

(a, b) Fluorescence images of IgG-CPP complexes localized to the endosome (a) or lysosome (b). White squares are shown as enlarged images. Scale bar is $10 \mu \mathrm{m}$.

ence of 2-iminothiolane to achieve crosslinking via thioether bonds (Fig. 1b). Circular dichroism demonstrated a positive cotton effect around $225 \mathrm{~nm}$, indicating that iodoacetylcolpep formed a triple-helical structure at $4{ }^{\circ} \mathrm{C}$. The melting temperature $\left(T_{\mathrm{m}}\right)$ for the triple helix of iodoacetyl-colpep was estimated to be $53.6^{\circ} \mathrm{C}$. This demonstrated that the triple helix of colpep was stable at body temperature.

FITC-IgG was treated with iodoaceyl-colpep or iodoacetyl$\boldsymbol{\beta} \mathbf{R 8}$ and 2-iminothiolane in aqueous solution at $\mathrm{pH}$ 8.5. The product was purified by gel filtration and concentrated by ultrafiltration. The conjugation of CPPs to FITC-IgG was confirmed by band shifts on SDS-PAGE under reduced conditions (data not shown). During the preparation of IgG-CPP complexes, we observed differences in the yields between colpep and $\boldsymbol{\beta} \mathbf{R 8}$ (Fig. 1c). The use of higher concentrations of $\boldsymbol{\beta} \mathbf{R} 8$ led to lower recovery of the desired product. This observation is consistent with a previous report that the conjugation of oligoarginine with antibodies tends to lead to aggregation. ${ }^{8)}$ 
Conversely, forming conjugates with colpep afforded higher and more consistent product recovery. These results demonstrate a clear efficiency advantage of using a triple-helical CPP to label IgG.

The uptake of CPP-conjugated FITC-IgG into HeLa cells was analyzed by confocal laser microscopy (Fig. 2a). Significant fluorescence was observed following treatment with both IgG-colpep and IgG- $\boldsymbol{\beta}$ R8. This indicated that the delivery of full-length $\operatorname{IgG}$ into cells was achieved by conjugation to CPPs. FACS analysis revealed that IgG-colpep was more efficiently taken into cells than IgG- $\boldsymbol{\beta} \mathbf{R} 8$ (Fig. 2b). In contrast to IgG-colpep, cellular uptake of IgG- $\boldsymbol{\beta}$ R8 was significantly neutralized by FBS pre-treatment for $24 \mathrm{~h}$ (Fig. 2c).

The intracellular distribution of CPP-conjugated IgG was analyzed by live cell imaging. Using endosomal staining using TRITC-transferrin, most of the fluorescence derived from antibodies colocalized with that from endosomes (Fig. 3a). In addition, both IgG-colpep and IgG- $\boldsymbol{\beta}$ R8 were also partly localized in lysosomes (Fig. 3b). These results indicate that the IgG-CPP complexes were taken up by cells via the endocytotic pathway and remained trapped in vesicles.

\section{DISCUSSION}

We investigated the delivery of full-length $\operatorname{IgG}$ into cells by CPPs. The previously reported triple-helical CPP was a heterotrimer whose preparation was accomplished by means of stepwise and regioselective disulfide bond-forming reactions. ${ }^{10)}$ In this report, we employed a homotrimeric CPP for more convenient preparation. In addition, one-pot conjugation of colpep to IgG was achieved using 2-iminothiolane as a crosslinking reagent. The recovered yield of IgG-colpep was higher than that of IgG- $\boldsymbol{\beta} \mathbf{R} \mathbf{8}$ because of the difference in aggregation tendency of the CPPs. Here we demonstrated a facile and higher yield method to conjugate full-length IgG and CPPs.

The efficacy of colpep for delivering $\operatorname{IgG}$ into cells was higher, and less impaired by serum, than that of $\boldsymbol{\beta} \mathbf{R 8}$ (Fig. 2 ). This result could be accounted for by lower binding of colpep to serum proteins compared with $\boldsymbol{\beta} \mathbf{R 8} .^{10)}$ However, both intracellular IgG-colpep and IgG- $\boldsymbol{\beta} \mathbf{R} 8$ were trapped in endosomes (Fig. 3). Although cargo molecules have known to be primarily taken up by CPP via endocytosis, direct membrane penetration is also considered an additional mechanism of uptake. ${ }^{12)}$ A theoretical model for the interaction of human immunodeficiency virus (HIV) Tat peptide (a well-known CPP) with membrane suggests the transient pore formation. ${ }^{13)}$ However, macromolecules such as proteins and antibodies are considered to be hard to pass these pores because of too large molecular size. Actually, smaller cargoes tend to be taken up by membrane transduction, while larger molecules are mainly trapped in endocytosis. ${ }^{14-16)}$ Our triple-helical cell-penetrating peptide probably could not form pores in the membranes. Some progresses in overcoming this drawback such as combination with membrane-disruptive liposomes, peptides or polymers have been made. ${ }^{17-19)}$

(Gly-Xaa-Yaa)-repeating peptides which we employed in this research form collagen-like triple-helical structure. We have revealed that triple-helical peptides are quite stable even in the gastrointestinal tract where many digestive enzyme work. ${ }^{20)}$ Therefore, triple-helical CPP conjugated to IgG is probably considered to be almost intact in endosomal compartment. We are now researching a novel triple-helical CPP with endosome-escaping properties.

Acknowledgments This work is supported by Waseda University Grant for Special Research Projects (2013A-043) and the collaboration between Mitsubishi Materials Corporation and Faculty of Science and Engineering, Waseda University.

Conflict of Interest The authors declare no conflict of interest.

\section{REFERENCES}

1) Zolot RS, Basu S, Million RP. Antibody-drug conjugates. Nat. Rev. Drug Discov., 12, 259-260 (2013).

2) Shim H. One target, different effects: a comparison of distinct therapeutic antibodies against the same targets. Exp. Mol. Med., 43, 539-549 (2011).

3) Heitz F, Morris MC, Divita G. Twenty years of cell-penetrating peptides: from molecular mechanisms to therapeutics. Br. J. Pharmacol., 157, 195-206 (2009).

4) Futaki S. Oligoarginine vectors for intracellular delivery: design and cellular-uptake mechanisms. Biopolymers, 84, 241-249 (2006).

5) Nakase I, Konishi Y, Ueda M, Saji H, Futaki S. Accumulation of arginine-rich cell-penetrating peptides in tumors and the potential for anticancer drug delivery in vivo. J. Control. Release, 159, 181-188 (2012).

6) Zhao Y, Lou D, Burkett J, Kohler H. Chemical engineering of cell penetrating antibodies. J. Immunol. Methods, 254, 137-145 (2001).

7) Morris MC, Depollier J, Mery J, Heitz F, Divita G. A peptide carrier for the delivery of biologically active proteins into mammalian cells. Nat. Biotechnol., 19, 1173-1176 (2001).

8) Niesner U, Halin C, Lozzi L, Günthert M, Neri P, Wunderli-Allenspach H, Zardi L, Neri D. Quantitation of the tumor-targeting properties of antibody fragments conjugated to cell-permeating HIV-1 TAT peptides. Bioconjug. Chem., 13, 729-736 (2002).

9) Takayama K, Tadokoro A, Pujals S, Nakase I, Giralt E, Futaki S. Novel system to achieve one-pot modification of cargo molecules with oligoarginine vectors for intracellular delivery. Bioconjug. Chem., 20, 249-257 (2009).

10) Yamazaki CM, Nakase I, Endo H, Kishimoto S, Mashiyama Y, Masuda R, Futaki S, Koide T. Collagen-like cell-penetrating peptides. Angew. Chem. Int. Ed. Engl., 52, 5497-5500 (2013).

11) Shinde A, Feher KM, Hu C, Slowinska K. Peptide internalization enabled by folding: triple helical cell-penetrating peptides. J. Pept. Sci., 21, 77-84 (2015).

12) Marschall AL, Frenzel A, Schirrmann T, Schüngel M, Dübel S. Targeting antibodies to the cytoplasm. MAbs, 3, 3-16 (2011).

13) Herce HD, Garcia AE. Molecular dynamics simulations suggest a mechanism for translocation of the HIV-1 TAT peptide across lipid membranes. Proc. Natl. Acad. Sci. U.S.A., 104, 20805-20810 (2007).

14) Youngblood DS, Hatlevig SA, Hassinger JN, Iversen PL, Moulton HM. Stability of cell-penetrating peptide-morpholino oligomer conjugates in human serum and in cells. Bioconjug. Chem., 18, 50-60 (2007).

15) Rinne J, Albarran B, Jylhava J, Ihalainen TO, Kankaanpaa P, Hytonen VP, Stayton PS, Kulomaa MS, Vihinen-Ranta M. Internalization of novel non-viral vector TAT-streptavidin into human cells. BMC Biotechnol., 7, 1 (2007).

16) Tünnemann G, Martin RM, Haupt S, Patsch C, Edenhofer F, Cardoso MC. Cargo-dependent mode of uptake and bioavailability of 
TAT-containing proteins and peptides in living cells. FASEB J., 20, 1775-1784 (2006).

17) El-Sayed A, Futaki S, Harashima H. Delivery of macromolecules using arginine-rich cell-penetrating peptides: ways to overcome endosomal entrapment. AAPS J., 11, 13-22 (2009).

18) Nakase I, Kobayashi S, Futaki S. Endosome-disruptive peptides for improving cytosolic delivery of bioactive macromolecules. Biopolymers, 94, 763-770 (2010).
19) Loughran SP, McCrudden CM, McCarthy HO. Designer peptide delivery systems for gene therapy. Eur. J. Nanomed., 7, 85-96 (2015).

20) Koide T, Yamamoto N, Yasui H. Fecal excretion of orally administered collagen-like peptides in rats: contribution of the triple-helical conformation to their stability. Biol. Pharm. Bull., 39, 135-137 (2016). 